\title{
TerraSAR-X precise orbit determination with real-time GPS ephemerides
}

\author{
M. Wermuth*, A. Hauschild, O. Montenbruck, R. Kahle \\ German Space Operation Center (DLR/GSOC), Oberpfaffenhofen, 82234 Wessling, Germany
}

Received 14 October 2011; received in revised form 7 March 2012; accepted 8 March 2012

Available online 16 March 2012

\begin{abstract}
For active and future Earth observation missions, the availability of near real-time precise orbit information is becoming more and more important. The latency and quality of precise orbit determination results is mainly driven by the availability of precise GPS ephemerides and clocks. In order to have high-quality GPS ephemerides and clocks available at real-time, the German Space Operations Center (GSOC) has developed the real-time clock estimation system RETICLE. The system receives data streams with GNSS observations from the global tracking network of the International GNSS Service (IGS) in real-time. Using the known station position, RETICLE estimates precise GPS satellite clock offsets and drifts based on the most recent available ultra rapid predicted orbits provided by the IGS. The clock offset estimates have an accuracy of better than $0.3 \mathrm{~ns}$ and are globally valid. The latency of the estimated clocks is approximately $7 \mathrm{~s}$ after the observation epoch.

Another limiting factor is the frequency of satellite downlinks and the latency of the data transfer from the ground station to the operations center. Therefore a near real-time scenario using GPS observation data from the TerraSAR-X mission is examined in which the satellite has about one ground station contact per orbit or respectively one contact in $90 \mathrm{~min}$. This test campaign shows that precise orbits can be obtained in near real-time. With the use of estimated clocks an orbit accuracy of better than $10 \mathrm{~cm}$ (3D-RMS) can be obtained. The evaluation of satellite laser ranging (SLR) observations shows residuals of $2.1 \mathrm{~cm}$ (RMS) for orbits using RECTICLE and residuals of $4.2 \mathrm{~cm}$ (RMS) for orbits using the IGS ultra rapid ephemerides and clocks products. Hence the use of estimated clocks improves the orbit determination accuracy significantly ( factor 2 ) compared to using predicted clocks.
\end{abstract}

(c) 2012 Published by Elsevier Ltd. on behalf of COSPAR.

Keywords: GPS, Precise orbit determination; Real time clock estimation; TerraSAR-X

\section{Introduction}

During the last two decades, the availability of geodetic grade GPS receivers on Earth observation missions and the development of reduced dynamic orbit determination (e.g. Wu et al., 1991) have made precise orbit determination (POD) accuracies of $10 \mathrm{~cm}$ or less (in a 3D RMS sense) possible. This has been shown for several low Earth orbiting (LEO) missions like TOPEX/Poseidon, Jason 1, Jason 2 (Cerri et al., 2010; Lemoine et al., 2010), CHAMP

\footnotetext{
* Corresponding author. Tel.: +49 8153282155; fax: +49 8153281450 .

E-mail addresses: martin.wermuth@dlr.de (M. Wermuth), andre. hauschild@dlr.de (A. Hauschild), oliver.montenbruck@dlr.de (O. Montenbruck), Ralph.kahle@dlr.de (R. Kahle).
}

(Reigber et al., 2002) or GRACE (Tapley et al., 2004a). The quality of the results is - among other factors like the quality of the GPS observations and the employed models - dependent on the accuracy of the employed GPS ephemerides and clocks. The International GNSS Service (IGS) provides a rapid GPS ephemerides product with a latency of $17 \mathrm{~h}$ and a final product with a latency of about 13 days (Dow et al., 2009).

In the future, availability of near real-time POD for applications like altimetry (Desai et al., 2010; Jayles et al., 2010), atmospheric limb sounding (König et al., 2002) or on-board navigation in real-time for formation flying and docking missions (e.g. D'Amico et al., 2012) will become more and more important. For real-time POD the IGS provides "ultra-rapid" GPS ephemerides, which 
contain a $24 \mathrm{~h}$ arc based on observations and a $24 \mathrm{~h}$ part based on orbit and clock predictions for the GPS satellites. Due to the prediction, those ephemerides are always available at real-time - at the cost of accuracy.

The Jet Propulsion Laboratory (JPL) has made the earliest contributions to real-time orbit and clock corrections. Already in 1996, JPL set up a receiver network covering the entire United States and computed real-time corrections based on the measurement streams of these receivers (Bertiger et al., 1998). This network was soon expanded to global coverage and has today about 100 stations. The derived orbit and clock corrections can be received by users on the Earth and even in space (Bar-Sever et al., 2004). In 2007, the IGS has launched a Real-Time Pilot Project in order to promote the development of freely accessible real-time products. Several institutions participate in this project and submit contributions for comparison and combination (Mervart et al., 2008; Collins et al., 2005; Hauschild and Montenbruck, 2009; Ge et al., 2010; Zhang et al., 2010; Laurichesse et al., 2010). Furthermore, several commercial providers offer real-time corrections (Dixon et al., 2006; Melgard et al., 2009; Leandro et al., 2011).

In order to have an in-house real-time solution available at the German Space Operations Center (GSOC) with higher accuracy, we have developed a real-time clock estimation (RETICLE) process. It is based on the orbits of the IGS ultra-rapid ephemerides but estimates clocks from observations almost in real-time, using a Kalman-filter approach and improving the accuracy of the ephemerides and clocks product. A similar concept using least squares estimation of near real-time clocks for IGS ultra-rapid orbits was already proposed (Bock et al., 2009).

To assess the performance using RETICLE products for near real-time POD, we have performed two test campaigns with the TerraSAR-X satellite involving almost one ground station contact per revolution. The real-time POD results are compared with results using the classical GPS ephemerides products. In addition, satellite laser ranging (SLR) observations are used to evaluate the accuracy of the POD results, as they are the only measurement type available on TerraSAR-X apart from GPS.

\section{TerraSAR-X Routine POD}

TerraSAR-X is a German radar satellite mission, which is realized as a public-private partnership between the German Aerospace Center (DLR) and EADS Astrium GmbH. Its observation technique for the acquisition of radar images of the Earth's surface is synthetic aperture radar (SAR) (Werninghaus and Buckreuss, 2010). The satellite is controlled by the mission operations segment (MOS) which is located at GSOC. Among the various tasks of the MOS is the provision of precise orbits of the spacecraft to the scientific users.

The TerraSAR-X satellite was launched on June 15th, 2007 on a Russian DNEPR rocket from Baikonur in Kazakhstan. Since then it is acquiring radar images of the Earth's surface with its main payload - a SAR instrument. The satellite weighs about $1300 \mathrm{~kg}$, is $5 \mathrm{~m}$ long and has a diameter of $2.4 \mathrm{~m}$. It is operated by GSOC at an orbit height of $515 \mathrm{~km}$ with an inclination of $97.44^{\circ}$. In order to enable repeat-pass interferometry, the satellite is flown at an Earth-fixed reference orbit, which repeats after 11 days with 167 revolutions. This requires - dependent on solar activity - roughly one orbit maintenance maneuver per week in year 2010 .

To achieve its mission objectives, the TerraSAR-X satellite is equipped with two on-board receivers. The MosaicGNSS receiver, developed by EADS/Astrium (Krauss et al., 2010) is a single frequency receiver with eight channels for tracking GPS satellites. It is mainly used for onboard navigation and timing. For the purpose of precise obit determination, the Integrated Geodetic Occultations and Ranging (IGOR) receiver (Rothacher et al., 2007) is used. It was developed by Broad Reach Engineering and provided by the German Research Centre for Geosciences (GFZ) and is a heritage of the space proven Blackjack receiver, which was employed on missions like CHAMP and GRACE. It is a geodetic grade dual-frequency receiver with 12 channels allocated for tracking GPS satellites for navigation. Additionally 4 channels are used for occultation observations, which is a secondary mission objective.

In order to process the radar images correctly, the knowledge of precise orbit positions is necessary. The POD is carried out by GSOC on a routine basis (Yoon et al., 2009). The process is based on a reduced dynamic orbit determination using the "GPS High precision Orbit determination Software Tools" (GHOST), which was developed at GSOC in cooperation with Delft University of Technology (e.g. Montenbruck et al., 2005). The POD is executed in two different operational chains. A rapid POD with short latency and reduced accuracy is used for a quick-look evaluation of the acquired SAR data takes. The science POD provides the best possible solution for the final processing of the radar scenes after a couple of days. All employed physical models are listed in Table 1 . They are identical for the rapid POD and the science POD and as well for the near real-time POD described below.

\subsection{Rapid POD}

The rapid POD serves the purpose of quick-look processing of acquired radar images. Its main focus is quick availability at the cost of reduced accuracy. The product generation process is driven by ground station contacts. During routine operations a single ground station (located in Weilheim/Germany and operated by GSOC) is sufficient for commanding and downlinking of housekeeping data (including GPS raw observations). This station has on average 4 contacts per day during which the GPS raw observation data is retrieved from the satellite. A rapid POD process is executed after each ground station contact. The required maximum latency for a rapid POD product is $19 \mathrm{~h}$. Using the IGS ultra-rapid GPS ephemeris products 
Table 1

GSOC processing standards for TerraSAR-X.

\begin{tabular}{|c|c|}
\hline Item & Description \\
\hline $\begin{array}{l}\text { GPS measurement } \\
\text { model }\end{array}$ & $\begin{array}{l}\text { Undifferenced ionosphere-free code and carrier phase observations; corrected for approximate receiver clock offset; } 0^{\circ} \text { cut-off } \\
\text { elevation w.r.t. horizon; Phase center offsets and variations of transmitter and receiver antennas; phase wind-up. }\end{array}$ \\
\hline $\begin{array}{l}\text { SLR measurement } \\
\text { model }\end{array}$ & $\begin{array}{l}\text { SLRF2005 coordinates; solid Earth and pole tides (McCarthy and Petit, 2004); GOT00.2 ocean loading;Marini \& Murray } \\
\text { tropospheric delay model (McCarthy and Petit, 2004). }\end{array}$ \\
\hline Gravitational forces & $\begin{array}{l}\text { GRACE Gravity model } 01 \text { (GGM01) degree/order100 (Tapley et al., 2004b); relativitysolid-earth tides; pole tide; ocean tides: } \\
\text { CSR 3.0 (Eanes and Bettadpur, 1996); luni-solar third body acceleration using analytical ephemerides }\end{array}$ \\
\hline $\begin{array}{l}\text { Non-gravitational } \\
\text { forces }\end{array}$ & $\begin{array}{l}\text { Jacchia-Gill atmospheric density model with daily } \mathrm{F}_{10.7} \text { and 3-hourly } \mathrm{K}_{\mathrm{p}} \text { values; Cannon ball solar radiation pressure model } \\
\text { with conical Earth shadow model (umbra, penumbra); Empirical accelerations in radial, along-track and cross-track direction } \\
\text { at } 10 \text { min intervals; Constant thrust values are estimated for maneuvers. }\end{array}$ \\
\hline Numerical integration & Variable-order variable step-size Adams-Bashforth multipstep method. \\
\hline Reference frames & $\begin{array}{l}\text { EME2000; IAU } 1976 \text { precession (Lieske model); IAU } 1980 \text { nutation (Wahr model); Earth orientation from IERS igs96p02 } \\
\text { solution; Spacecraft body frame orientation relative to EME2000 based on star sensor attitude determination. }\end{array}$ \\
\hline Estimation & Batch least-squares. \\
\hline
\end{tabular}

(including clocks) for the POD, a latency of only 3-9 h is achieved, as these products arrive with a latency of $3 \mathrm{~h}$ at an update rate of $6 \mathrm{~h}$. During each run an orbit arc of at least $12 \mathrm{~h}$ is computed in order to include all new GPS data and an overlap of several hours to the previous run for consistency checks.

The required accuracy for rapid POD results is $2 \mathrm{~m}$ (3DRMS) to ensure a proper quick-look processing of radar images. Comparing rapid POD results to the science POD results, which are considered to be much more accurate, one can asses the accuracy of the rapid POD results. Fig. 1 shows the difference of several overlapping rapid POD arcs on June 14th 2010 with the corresponding science orbit. It can be seen, that the global difference is about $11 \mathrm{~cm}$ (3D-RMS). Hence the accuracy requirement is fulfilled, which is confirmed by SLR observations (see Section 5 or Yoon et al., 2009), but at the start and end of each rapid POD arc errors of up to $0.5 \mathrm{~m}$ caused by edge effects are visible.

\subsection{Science $P O D$}

The required accuracy for the science orbit product is $20 \mathrm{~cm}$ (3D-RMS). This requirement is driven by the accuracy needs of repeat-pass interferometry (Bamler and Hartl, 1998). This is an acquisition technique, where SAR-images of the same scene under the same geometry, but different acquisition time (e.g. second data-take is taken during the next cycle of 11 days) are compared.

In order to ensure the availability of all input data and auxiliary data - and thus the best possible accuracy - the science POD is executed daily with a latency of 5 days. GPS ephemerides and high-rate clocks (30 s sampling rate), from the rapid product series of the Center for Orbit Determination in Europe (CODE) analysis center (Dach et al., 2009) of the IGS are employed. The daily orbits are computed as $30 \mathrm{~h}$ arcs centred at noon, to provide an overlap of $6 \mathrm{~h}$ to the previous orbit product for quality assessment. Comparisons with satellite ranging data (see Section 5) and independent orbit solutions of other institutions (Yoon et al., 2009) lead to the conclusion, that an accuracy of
$5 \mathrm{~cm}$ (3D-RMS) is achieved. The accuracy requirement of $20 \mathrm{~cm}$ is met.

\section{Real-time clock estimation (RETICLE) system}

DLR's real-time clock estimation (RETICLE) system provides corrections for satellite clock offsets of all GPS satellites with a latency of a few sec with respect to the measurement epoch (Hauschild and Montenbruck, 2008). The system processes dual-frequency pseudorange and carrierphase measurements from a global real-time tracking network depicted in Fig. 2. The network comprises mainly stations selected from the IGS and is supplemented by stations from the cooperative network for GIOVE observation (GONGO) (Montenbruck et al., 2011). A Kalmanfilter estimates satellite clock offset corrections based on predicted ultra-rapid orbits from IGS. The filter state comprises a satellite clock offset and a drift parameter for each satellite, a station clock offset and a tropospheric zenith delay parameter for each station and float ambiguities for all satellites tracked by each station. The estimated clocks are consistent with the ionosphere-free combination of the $\mathrm{P}(\mathrm{Y})$ code on the L1 and L2 frequency and are valid for the whole constellation.

Fig. 3 illustrates the data flow and the filter update cycle of the RETICLE system. The real-time data streams are received via the internet and contain measurements and broadcast ephemerides. The ultra-rapid orbits from IGS are retrieved via FTP at regular intervals to assure that the most recent predictions are used for the clock estimation process. Reference station positions are retrieved from IGS SINEX (Solution (Software/technique) INdependent EXchange Format, see: IERS, 2006 (International Earth Rotation and Reference System Service)) files and information about antenna phase center offset and variations for satellite and ground station antennas are taken from IGS ANTEX (Antenna Exchange Format, see: Schmid et al., 2007) files. Differential code biases from CODE are used for modelling the ionosphere-free combination consistently for stations which provide the C/A pseudo range instead of $\mathrm{P}(\mathrm{Y})$ code on $\mathrm{L} 1$. The station movements due to Earth 

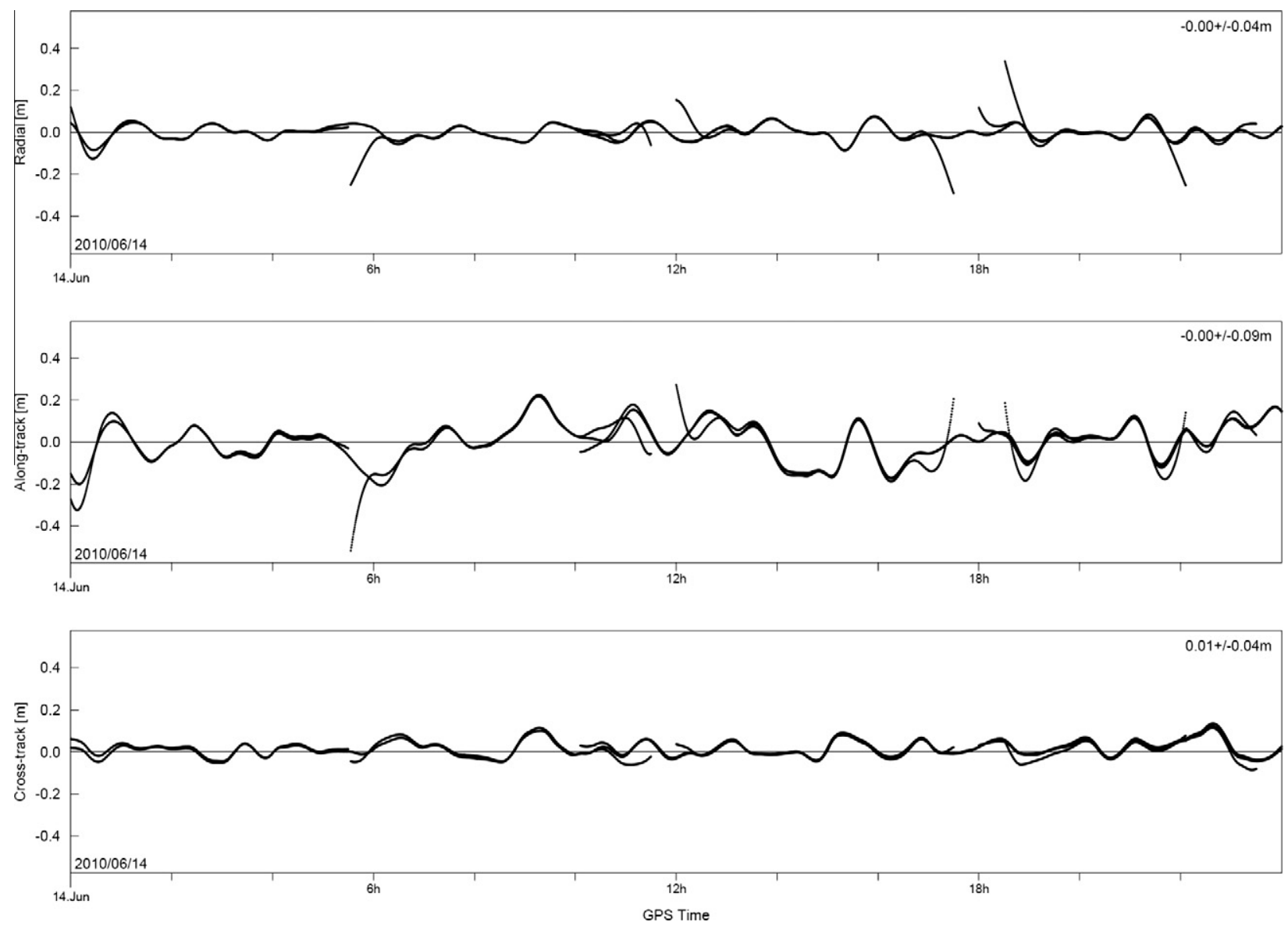

Fig. 1. Comparison of several overlapping rapid POD arcs with a science orbit on Jun. 14, 2010.

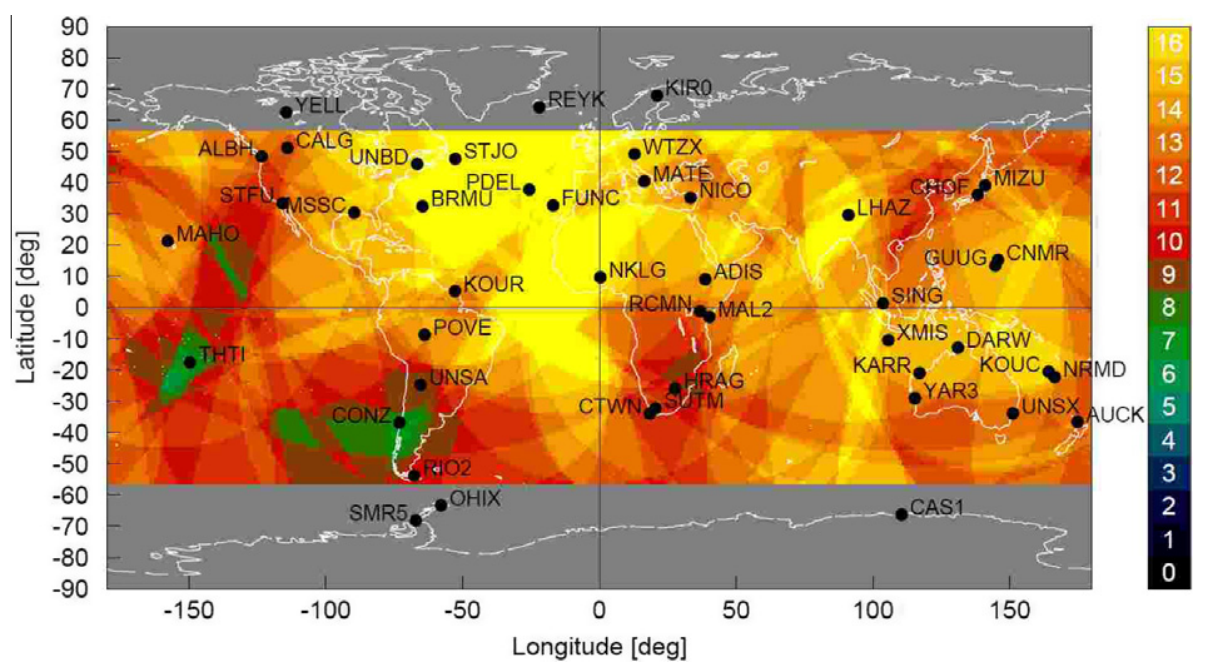

Fig. 2. Global real-time tracking network for clock estimation with RETICLE. The color-code indicates the number of stations, which can track a satellite simultaneously at a given position.

tides are modelled according to the IERS conventions (McCarthy and Petit, 2004). Earth-orientation parameters (EOP) for this model are retrieved from IGS.

A Kalman-filter update cycle is performed every $10 \mathrm{~s}$ and starts with a pre-processing step to detect corrupt measure- ments and exclude them from the measurement update. Observations which violate predefined minimum thresholds for satellite elevation $\left(10^{\circ}\right)$ and carrier-to-noise density ratio $(25 \mathrm{~dB} \mathrm{~Hz})$ are edited. Outliers of pseudo range and carrierphase measurements and cycle-slips are identified during 


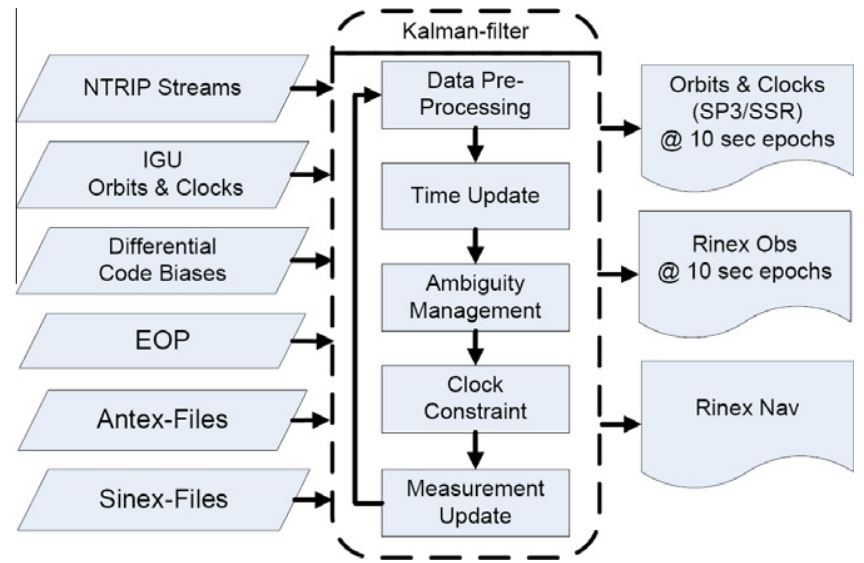

Fig. 3. Data flow and Kalman-filter update cycle of the RETILCE system.

a-priori residual screening. In the next step, the state vector is predicted from the previous epoch to the current epoch. Then the information about the ambiguities in the filter state is updated to handle changes in satellite visibility or cycle slips. If a receiver has lost track on a satellite, the corresponding ambiguity must be removed from the state vector. If a new satellite has been acquired by a receiver, the ambiguity must be initialized based on the difference between pseudo range and carrier-phase measurements. The same initialization is also performed if a cycle-slip has been detected to restart the estimation of the new float ambiguity. A clock constraint is applied in the following step to keep the mean offset of the estimated clocks weakly constrained to the mean of the predicted satellite clocks. Finally, the measurement update is performed using all pseudo range and carrier-phase measurements, which have passed the data pre-processing.

RETICLE's clock estimates can be retrieved by users in different formats. For near real-time users, the estimated clocks are logged together with the orbit predictions in SP3 files. The orbit positions are adopted from the IGU products and interpolated to the match the RETICLE sampling rate. These files are provided for download from an FTP $^{1}$ server with a latency of $5 \mathrm{~min}$. Users with demands for lower latencies can receive real-time streams from a dedicated server. These streams provide the clock and orbits as correction values to the current broadcast ephemerides in the new RTCM3 state-space-representation format (RTCM, 2011). In addition to orbits and clocks, RETICLE logs all observations in RINEX observation files (Gurtner and Estay, 2007) and provides the most recent broadcast ephemerides in daily RINEX navigation files and as RTCM3 stream (RTCM, 2011).

\section{Near real-time campaign with TerraSAR-X}

In order to demonstrate the performance of POD based on RETICLE GPS products, two short test campaigns

\footnotetext{
${ }^{1}$ The FTP-Server can be accessed under ftp.gsoc.dlr.de - contact the author for login details.
}

were carried out with TerraSAR-X. The first one took place from April 26th to April 28th, and the second one from June 11th to June 13th in 2010. The basic idea was to process GPS observation data with the same strategy and software as employed for the rapid POD chain, but with RETICLE GPS ephemerides. In order to create a near-real time environment, one ground station contact per orbit was planned in advance. Immediately after the arrival of down-linked GPS observation data, one near real-time orbit product was computed. By comparison with rapid and science POD results the latency and accuracy of the POD results from the two campaigns could be evaluated.

As mentioned above, the ground-station in Weilheim observes only 4 passages per day. Hence additional ground station contacts had to be ordered from near polar ground stations. Fig. 4 shows, that with the Norwegian ground station on Svalbard, and the Antarctic DLR ground-station in O'Higgins, almost every orbit could be covered.

Fig. 5 shows the visibility plot for the first campaign and Fig. 6 for the second test campaign. Solid blocks indicate a high-elevation pass, while empty blocks indicate a low-elevation pass, with an elevation below $10^{\circ}$. Low-elevation passes are normally not scheduled. The circles indicate the passes that were actually ordered. Several passes that failed are crossed out. During the first campaign, all O'Higgins passes failed due to ground-station maintenance activities. Due to network problems, during the second test campaign, the data from 4 passes arrived too late to be considered near real-time. Altogether 75 ground station contacts could be used for this study - 38 from Svalbard, 24 from Weilheim (which corresponds to routine operations) and 13 from O'Higgins.

On average the data was available at GSOC after $55 \mathrm{~min}$. The pre-processing took on average additional $23 \mathrm{~min}$, which mainly consisted of polling operations between different local networks. The latency for the availability of RETICLE GPS Ephemerides was negligible, as they were provided in real-time and thus were available before the GPS observations. The actual POD process took 5 min (for processing a $12 \mathrm{~h}$ arc), so that on average, the total time from ground-station contact to finished orbit products was $1 \mathrm{~h} 23 \mathrm{~min}$. The individual product latencies show dispersion between $50 \mathrm{~min}$ and $2 \mathrm{~h}$ (see Fig. 7) - in some cases even more. The source of these outliers lies in data-handling and has been identified and counter measures were taken since then. This means with one groundstation contact every $90 \mathrm{~min}$, on average the time passed since the data acquisition is between $1.5 \mathrm{~h}$ and $3 \mathrm{~h}$, when the POD is finished.

\section{Evaluation of the results}

In order to asses the quality of RETICLE clocks the Signal-in-Space Range Error (SISRE) is computed. The quality assessment of near real-time POD results in near real-time is difficult, as - apart from SLR observations, 

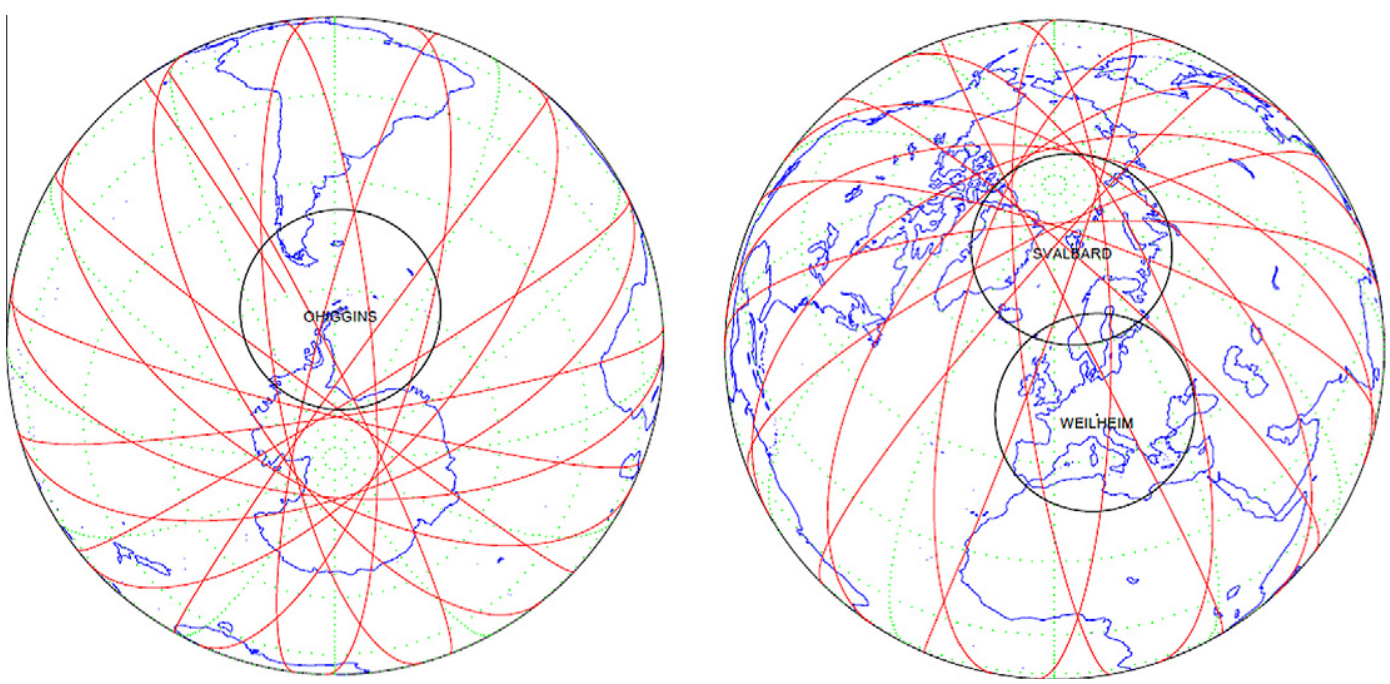

Fig. 4. Visibility of Weilheim, Svalbard and O'Higgins ground-stations (ground-track lines indicate the orbit of one day).
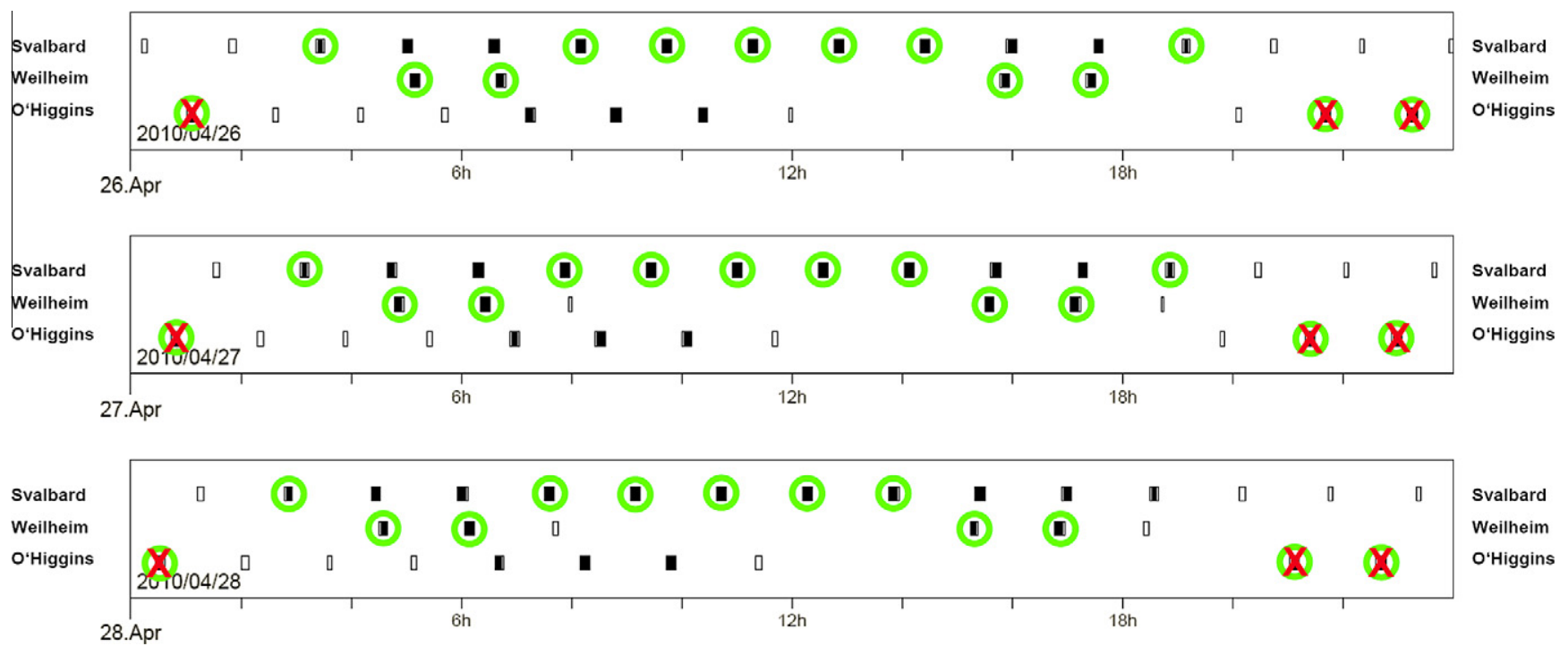

Fig. 5. Visibility of ground-stations and planned contacts for test campaign 1 (Apr 26-28 2010).

which are not available in real-time - no external and independent data source exists for TerraSAR-X. Thus SLR observations can only be used for evaluation in post processing. The same holds for a comparison with science orbits, which can give at least a rough impression on the quality of near real-time and rapid orbits. Overlaps to the previous arc and GPS residuals cannot provide an absolute quality measure, but are evaluated by the automated system in order to minimize the delivery of faulty orbit products. Finally a failure case study was carried out to show the impact of data loss.

\subsection{Signal-in space range error (SISRE)}

The quality of different GPS ephemerides and clocks products can be assessed by a direct comparison of the products. This can however not provide an absolute value of position and clock accuracy. The SISRE (e.g. Warren and Raquet, 2003) is a coarse value for the combined position error in line of sight and the clock error. Fig. 8 shows the daily SISRE values for both test campaigns. The daily values vary between $5.7 \mathrm{~cm}$ and $6.9 \mathrm{~cm}$, which is representative for longer RETICLE time series as well (Hauschild and Montenbruck, 2009). This shows that the RETICLE clock estimates are better than $0.3 \mathrm{~ns}$.

\subsection{GPS residuals}

In Fig. 9 the GPS residuals for one near real-time $12 \mathrm{~h}$ POD are shown. The last $1.5 \mathrm{~h}$ represent the update using new data obtained during the last ground-station contact. It can be seen, that the quality does not decrease in the updated part of the orbit arc. The pseudo range residuals show a standard deviation of $56 \mathrm{~cm}$, while the residual of 

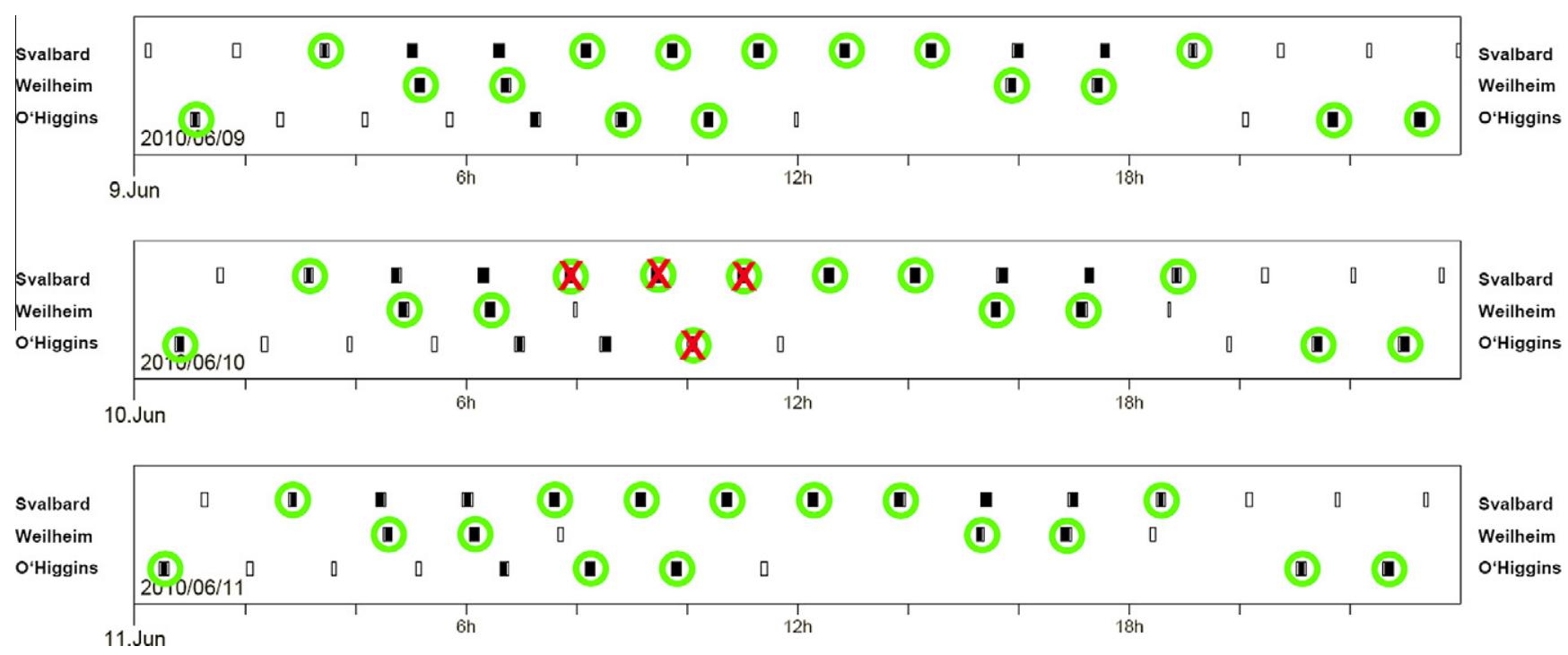

Fig. 6. Visibility of ground-stations and planned contacts for test campaign 2 (Jun 9-11 2010).

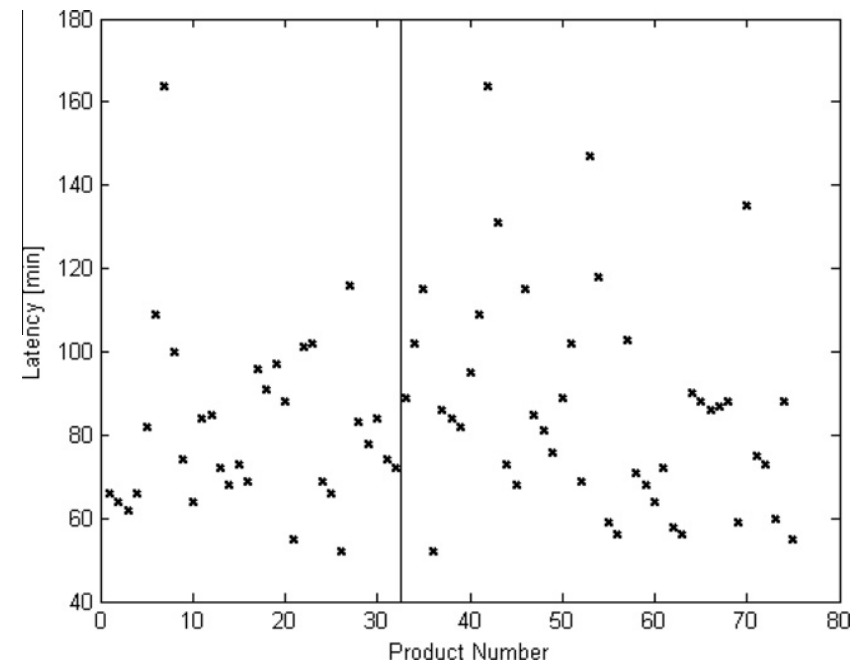

Fig. 7. Product latency (test campaign 1: \#1-\#32, test campaign 2: \#33 \#75).

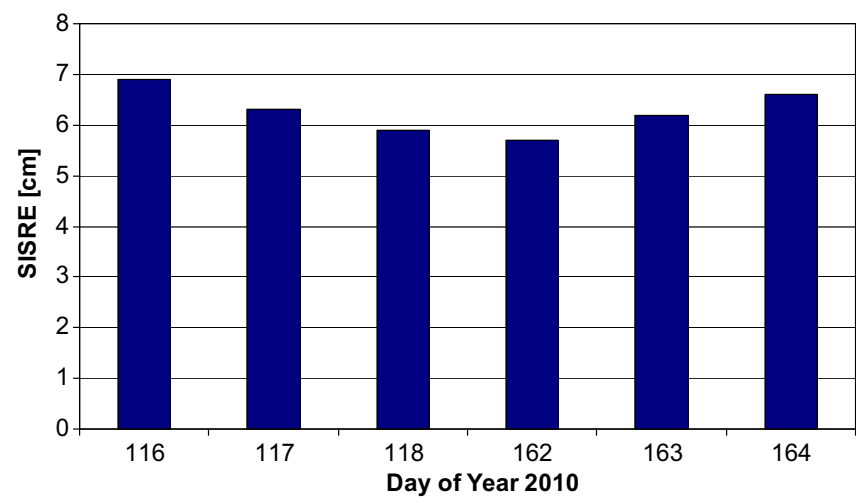

Fig. 8. Daily signal-in-space range errors for both test campaigns.

the carrier phase observations have a standard deviation of $6 \mathrm{~mm}$. In comparison, the standard deviation of carrier phase residuals for rapid orbits is at about $40 \mathrm{~mm}$, and for science orbits at $3-4 \mathrm{~mm}$. The a posteriori residuals are no absolute quality criteria, but this comparison shows, that the accuracy of the near real-time POD is much better, than that of the rapid POD, but does not reach the quality of the science POD.

\subsection{Comparison with science $P O D$}

The 75 single near real-time POD arcs have been compared with the corresponding science POD arcs. The 3D-RMS differences of each individual product are shown in Fig. 10. The overall difference over both test campaigns is $2.6 \mathrm{~cm}$ (3D-RMS). A group of five outliers with an RMS difference of about $5 \mathrm{~cm}$ can be identified. These outliers are caused by an undetected error in the raw GPS data. It appears in five POD arcs, due to the large overlap between products. This error has been detected during the science POD processing and manually removed. But an automated real-time process can obviously not detect all deficiencies in the GPS observations.

The differences for one arc are exemplarily shown in Fig. 11. Again the last $1.5 \mathrm{~h}$ represent the update part since the last ground-station contact. If only this part is considered, the RMS difference to the science POD rises to $3.5 \mathrm{~cm}$. A closer look on the update part shows deterioration only during the last $30 \mathrm{~min}$ of a product. Taking into account, that the GPS residuals do not show the same deterioration, the higher RMS can be explained by edge effects. These edge effects can often be observed in reduceddynamic POD and are for example even more prominent in the rapid POD (see Fig. 1).

Considering, that the accuracy of the science orbit was estimated to be well below the $10 \mathrm{~cm}$ level (3D-RMS) (see Yoon et al., 2009) or rather at the $5 \mathrm{~cm}$ level as suggested 

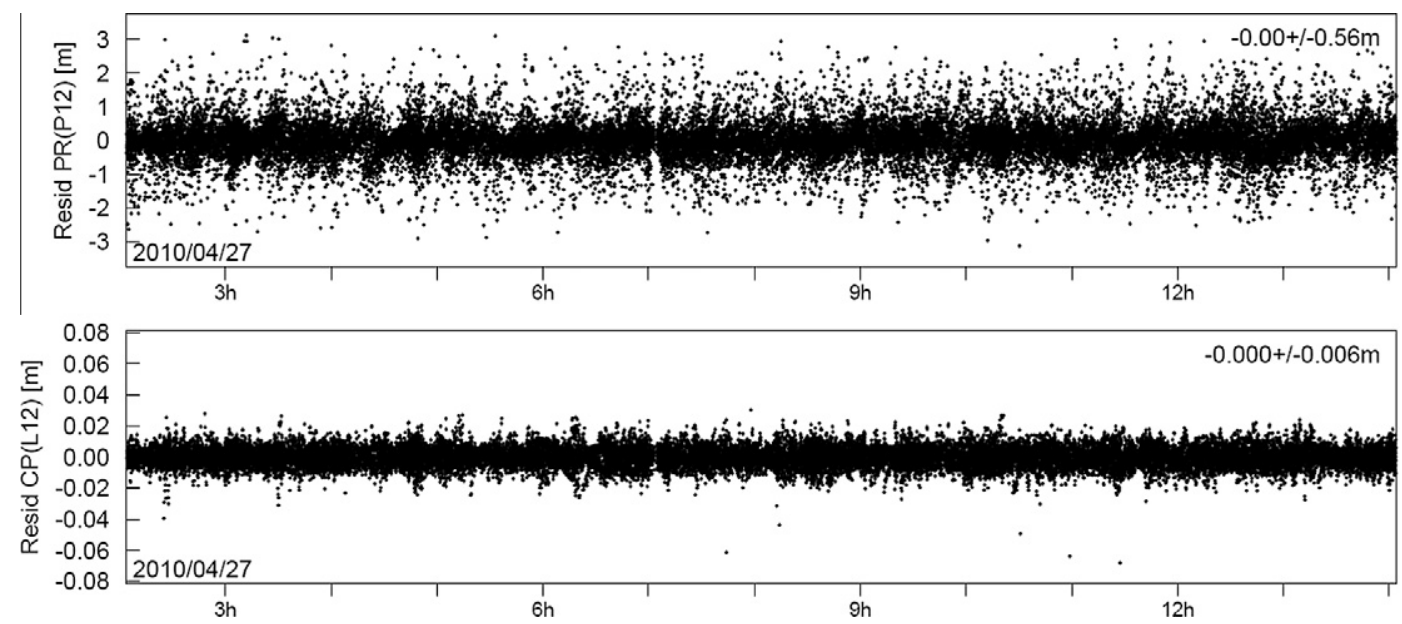

Fig. 9. Residuals of the ionosphere free linear combinations P12 and L12 for a $12 \mathrm{~h}$ near real-time POD arc (from 2:05 to 14:05, Apr. 27, 2010).

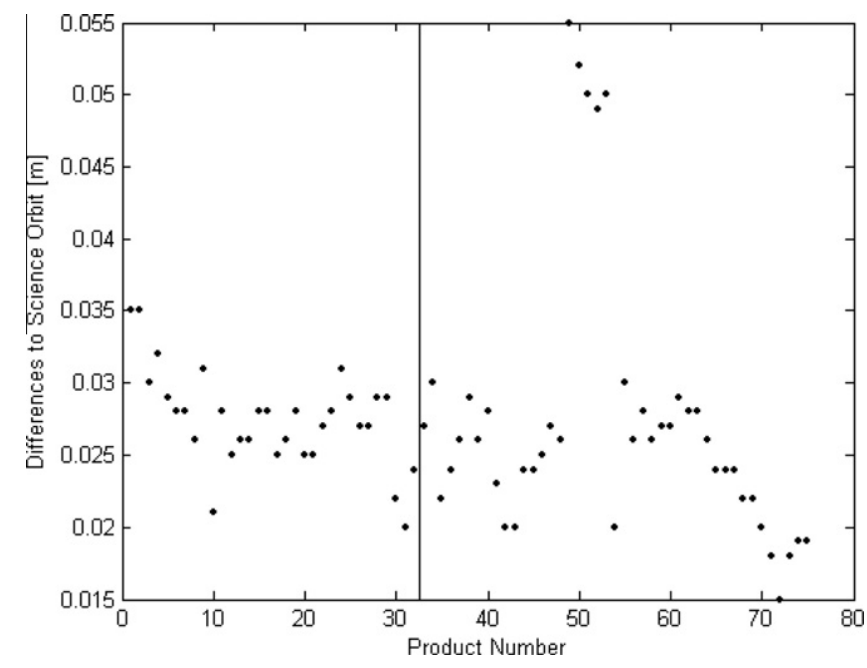

Fig. 10. Difference between near real-time POD arcs and science orbits (test campaign 1: \#1-\#32, test campaign 2: \#33 - \#75).

by the SLR residuals (see below), one can state, that the accuracy of the near real-time POD is as well below $10 \mathrm{~cm}$. Compared to that, the difference between rapid POD and science POD is at the order of $11 \mathrm{~cm}$.

\subsection{Validation with SLR observations}

As external quality check, satellite laser ranging observations are evaluated. During the 3 days of the first test campaign 693 SLR normal point observations from 9 different SLR stations are available. The corresponding residuals are shown in Fig. 12. For the second campaign only 408 observations from 4 different stations are available. The global RMS of the SLR observations is $2.1 \mathrm{~cm}$. For the science POD of the same period the SLR residuals show an RMS of $2.1 \mathrm{~cm}$ (generally SLR residuals for science POD are below $2 \mathrm{~cm}$ ), and for the rapid POD, the RMS is $4.2 \mathrm{~cm}$. This confirms that the accuracy of the near real-time POD is much better than that of the rapid POD, and almost reaches that of the science POD.

\subsection{Failure case study}

Operational environments are never perfect, and data can be lost on the path from spacecraft to the operations center via the ground-station. When using different ground-stations the data from one pass can arrive at the operations center after the following pass originating from another ground-station. In the operational system for TerraSAR-X, the product generation for the rapid orbit product would wait until the data gap was filled - or inform an operator if a certain latency threshold is exceeded. In an automated near real-time environment, there is no time to wait for operator interference. Hence we have performed a failure case study, to asses the impact of a lost pass. A gap was introduced to the GPS data from $3 \mathrm{~h}$ to $1.5 \mathrm{~h}$ before the end of the data. As can be seen in Fig. 13, the along-track error rises to $10 \mathrm{~cm}$ during the data-gap, but recovers again towards the end of a product. This shows that data gaps significantly disturb the solution, but as long as the data gaps do not include several revolutions have no serious impact.

\subsection{Summary}

It has been shown in this study, that a near real-time POD using RETICLE GPS products reached an accuracy of better than $10 \mathrm{~cm}$ (3D-RMS) for the TerraSAR-X campaign. The latency of the POD process was on average less than 90 min after data downlink. It became clear as well, that the latency of the near real-time POD process is mainly dependent on data availability after downlink, while the latency of the operational products is dependent on the availability of the GPS ephemerides. The latency and accuracy of the three POD products discussed in this paper are summarized in Table 2 .

\section{Conclusion and outlook}

This study has shown that the results obtained using RETICLE clocks are significantly more accurate than 

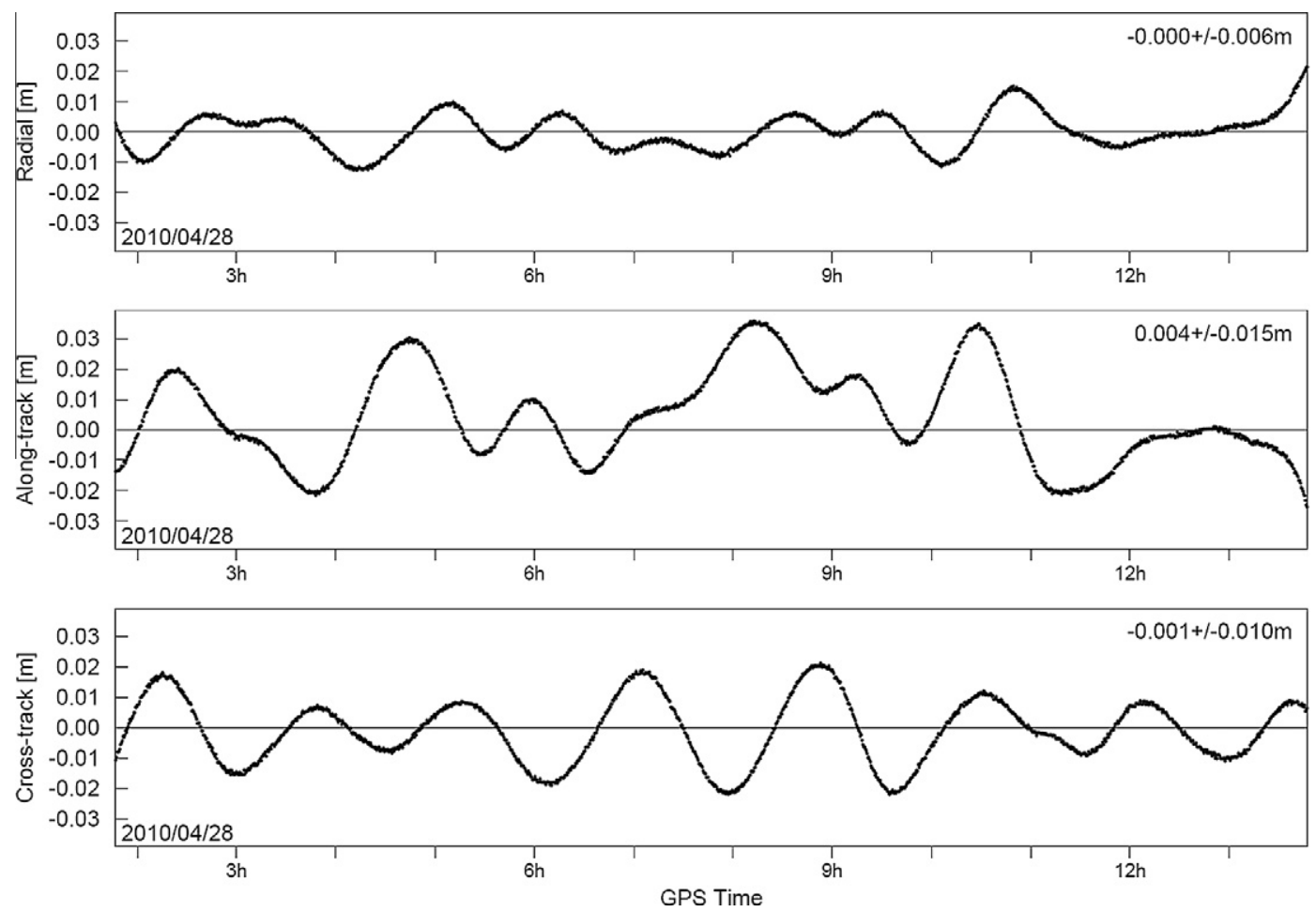

Fig. 11. Comparison of near real-time and science orbits (30s sampling rate).
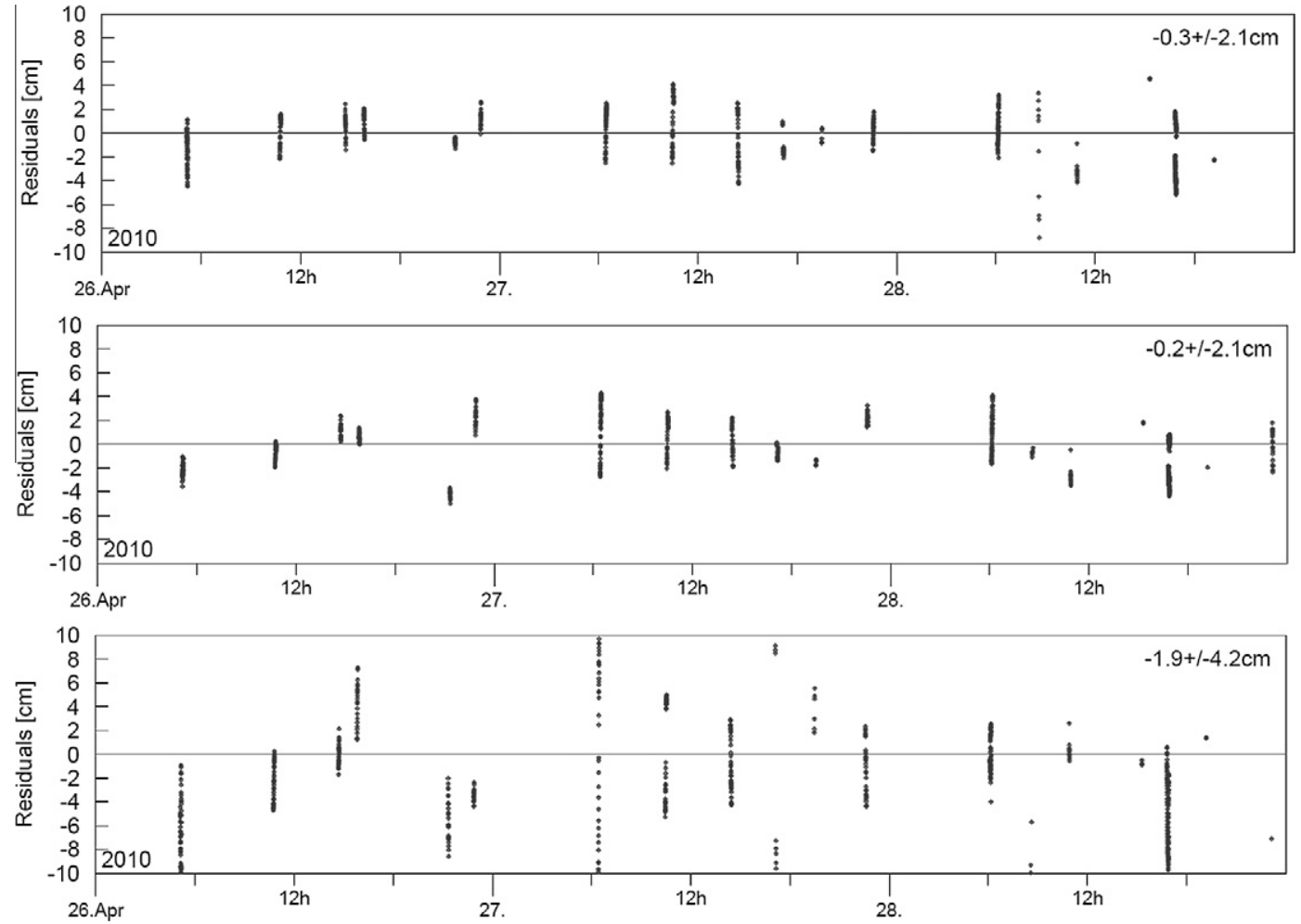

Fig. 12. SLR-Residuals for the near real-time (top), science (middle) and rapid (bottom) orbit products of test campaign 1.

those obtained using IGS ultra rapid orbits, although both have similar levels of accuracy for positions and clocks. This can be explained by a look on the sampling rate in
Table 2. The IGS ultra-rapid products have a sampling rate of $15 \mathrm{~min}$. The orbits of the GPS satellites are smooth enough to be interpolated over $15 \mathrm{~min}$ without large losses 

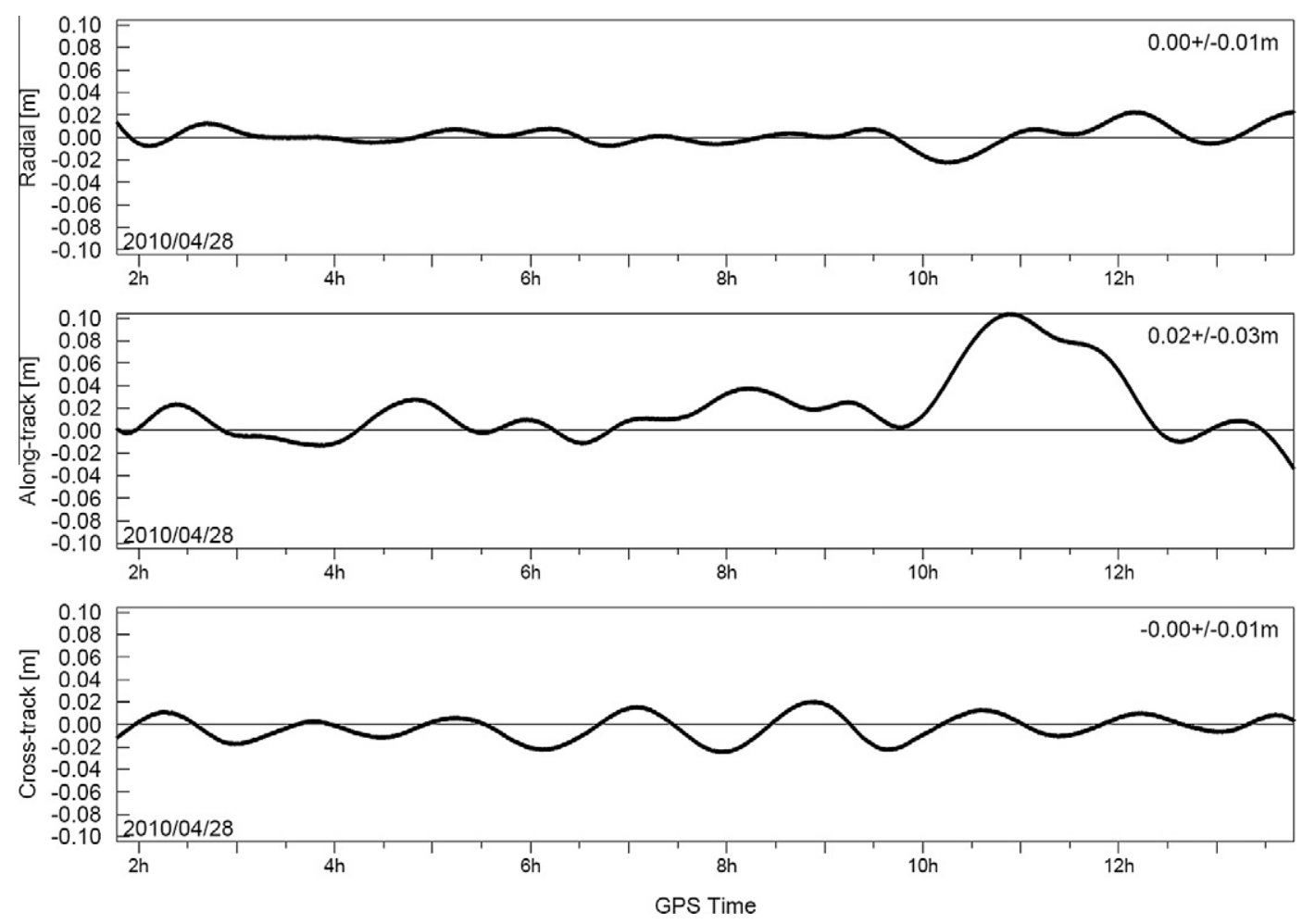

Fig. 13. Comparison of near real-time and science orbits (failure case).

Table 2

Latency and accuracy of different GSOC POD products for TerraSAR-X.

\begin{tabular}{llll}
\hline POD type & Science & Rapid & real-time \\
\hline Latency & several days & $3-9 \mathrm{~h}$ & $1.5-3 \mathrm{~h}$ \\
POD accuracy (3D) & $<5 \mathrm{~cm}$ & $<20 \mathrm{~cm}$ & $<10 \mathrm{~cm}$ \\
GPS orbit product & CODE rapid & IGS ultra-rapid & IGS ultra-rapid (resampled) \\
GPS clock product & CODE rapid & IGS ultra-rapid & RETICLE \\
GPS position accuracy & $<5 \mathrm{~cm}$ & $<5 \mathrm{~cm}$ (observed half) $\sim 10 \mathrm{~cm}$ (predicted half) & $\sim 10 \mathrm{~cm}$ \\
GPS Clocks Accuracy & $<0.1 \mathrm{~ns}$ & $\sim 0.2 \mathrm{~ns}$ (observed half) $\sim 5 \mathrm{~ns}$ (predicted half) & $<0.3 \mathrm{~ns}$ \\
GPS Clocks Sampling rate & $30 \mathrm{~s}$ & $15 \mathrm{~min}$ & $10 \mathrm{~s}$ \\
\hline
\end{tabular}

in accuracy. But the clock drift over that time span is so large, that interpolated clocks are suffering. Hence the sampling rate of $10 \mathrm{~s}$ of the RETICLE clocks is a significant advantage.

For future missions, where a near real-time availability of precise orbits becomes a requirement, the latency can be improved either by a ground segment design, with more frequent ground-station contacts and less transfer time (e.g. optimized polling between different networks) or by performing an onboard POD. Some of the delays in data-handling detected during the two test campaigns have already been optimized for a quicker data transfer between the satellite and the operations center. It has become clear, that near-polar ground stations near both poles are necessary to guarantee at least one contact during every revolution.

The real-time clock estimation filter will be operated on a routine basis, and thus GPS products with good quality will be available in real-time.

\section{Acknowledgements}

Precise GPS ephemerides for the precise orbit determination of TerraSAR-X are obtained from the Center for Orbit Determination in Europe (cf. Dach et al. 2009). The IGS ultra rapid ephemerides for rapid orbit determination are obtained from the International GNSS Service (cf. Dow et al., 2009). Satellite laser ranging measurements are provided by the International Laser Ranging Service (cf. Pearlman et al. 2002). The support of these institutions is gratefully acknowledged.

\section{References}

Bamler, R., Hartl, P., Synthetic aperture radar interferometry, Inverse Problems 14, R1-R54. http://dx.doi.org/10.1088/0266-5611/14/4/001, 1998.

Bar-Sever, Y.E., Young, L., Stocklin, F., et al., The NASA global differential GPS system (GDGPS) and the TDRSS augmentation service for satellites (TASS). In: Proceedings of ESA's 2nd Workshop 
on Navigation Equipment, Noordwijk, The Netherlands, WPP 239, December 2004.

Bertiger, W.I., Bar-Sever, Y.E., Haines, B.J., et al. A real-time wide area differential GPS system. J. Inst. Navig. 44 (4), 433-447, 1998.

Bock, H., Dach, R., Yoon, Y., et al., GPS clock correction estimation for near real-time orbit determination applications. Aerosp. Sci. Technol. 13 (7), 415-422. doi: 10.1016/j.ast.2009.08.003, 2009.

Cerri, L., Berthias, J.P., Bertiger, W.I., et al., Precision orbit determination standards for the Jason series of altimeter missions, Marine Geod. 33 (S1), 379-418, 2010.

Collins P., Gao, Y., Lahaye, F., et al., Accessing and processing realtime GPS corrections for precise point positioning - some user considerations. In: Proceedings of ION GNSS 18th international technical meeting of the satellite division, Long Beach, CA, US, 2005.

Dach, R., Brockmann, E., Schaer, S, et al., GNSS processing at CODE: status report. J. Geod. 83 (3-4), 353-365. doi: 10.1007/s00190-0080281-2, 2009.

D’Amico, S., Ardaens, J.-S., Larsson, R., Spaceborne autonomous formation flying experiment on the PRISMA mission; AIAA guidance, navigation, and control conference, 8-11 Aug. 2011, Portland, USA. In print: J. Guid. Control Dynamics. http://dx.doi.org/10.2514/ 1.55638, 2012.

Desai, S.D., Bertiger, W., Dorsey, A., et al. GPS-based precise orbit determination in near-real time for operational altimetry. Dv. Astron. Sci. 137, 593-604, 2010.

Dixon, K, StarFire: a global SBAS for sub-decimeter precise point positioning. In: Proceedings of ION GNSS 2006, 26-29 Sept. 2006, Fort Worth, TX, USA, 2286-2296, 2006.

Dow, J., Neilan, R.E., Rizos, C. The international GNSS service in a changing landscape of global navigation satellite systems. J. Geod. 83 (3-4), 191-198, 2009.

Ge, M., Chen, J., Douša, J., et al., A computationally efficient approach for estimating high-rate satellite clock corrections in realtime, GPS Solut. 16 (1):9-17. doi: 10.1007/s10291-011-0206-z, 2010.

Gurtner, W., Estey, L., RINEX - The Receiver Independent Exchange Format, Version 3.00, Nov 28, 2007, available online: http:// ftp.igs.org/igscb/data/format/rinex300.pdf, 2007.

Eanes, R.J., Bettadpur, S.V., The CSR 3.0 global ocean tide model, Center for Space Research, Techn. Memorandum, CSR-TM-96-05, 1996.

Hauschild, A., Montenbruck, O., Real-time clock estimation for precise orbit determination of LEO-satellites. In: Proceedings ION-GNSS2008 Conference, 581-589, A2b4, 16-19 Sept. 2008; Savannah, Georgia, 2008.

Hauschild, A., Montenbruck, O., Kalman-filter-based GPS clock estimation for near realtime positioning. GPS Solut. 13:173-182. doi: 10.1007/s10291-008-0110-3, 2009.

IERS, SINEX - Solution (Software/technique) INdependent EXchange Format Version 2.02 (December 01, 2006), available online: http:// www.iers.org/IERS/EN/Organization/AnalysisCoordinator/SinexFormat/sinex cont.html, 2006.

Jayles, C., Chauveau, J.P., Rozo, F. DORIS/Jason-2, better than $10 \mathrm{~cm}$ on-board orbits available for near-real-time altimetry. Adv. Space Res. 46 (12), 1497-1512, 2010.

König, R., Zhu, S., Neumayer, K.H., et al., CHAMP rapid orbit determination for GPS atmospheric limb sounding, Adv. Space Res. 30 (2), 289, 293, 2002.

Krauss, P.A., Kühl, C., Heim, J., et al., New applications for navigation receivers in space, satellite navigation technologies and european workshop on GNSS signals and signal processing (NAVITEC), 2010 5th ESA workshop on, 1-7, Dec. 8-10, 2010. doi: 10.1109/NAVITEC.2010.5707980, 2010.

Laurichesse, D., Mercier, F., Berthias, J.P., Real Time PPP with undifferenced integer ambiguity resolution, experimental results. In:
Proceedings of the ION GNSS 2010, 2534-2544, Portland, Oregon, 2010.

Leandro, R., Landau, H., Nitschke, M., et al., RTX Positioning: The Next Generation of cm-accurate Real-time GNSS Positioning, Proceedings of the ION GNSS 2011, 1460-1475, Portland, Oregon, 2011.

Lemoine, F.G., Zelensky, N.P., Chinn, D., et al. Towards development of a consistent orbit determination, TOPEX/Poseidon, Jason-1, Jason-2. Adv. Space Res. 46 (12), 1513-1540, 2010.

McCarthy, D.D., Petit, G., IERS Conventions 2003, IERS Technical Note 32, Frankfurt am Main: Verlag des Bundesamts für Kartographie und Geodäsie 127 pp., paperback, ISBN 3-89888-884-3, 2004.

Melgard, T., Vigen, E., de Jong, K., et al., G2-The first realtime GPS and GLONASS precise orbit and clock service. In: Proceedings of ION GNSS 2009, 1885-1891, 22-25 Sept 2009, Savannah, GA, USA, 2009.

Mervart, L., Lukes, Z., Rocken, C., et al., Precise point positioning with ambiguity resolution in realtime. In: Proceedings of ION GNSS 2008, 397-407, 16-19 Sept 2008, Savannah, GA, USA, 2008.

Montenbruck, O., van Helleputte, T., Kroes, R., et al., Reduced dynamic orbit determination using GPS code and carrier measurements. Aerosp. Sci. Technol. 9/3, 261-271. http://dx.doi.org/10.1016/ j.ast.2005.01.003, 2005.

Montenbruck, O., Hauschild, A., Hessels, U. Characterization of GPS/ GIOVE sensor stations in the CONGO network. GPS Solut. 15 (3), 193-205. doi: 10.1007/s10291-010-0182-8, 2011.

Pearlman, M.R., Degnan, J.J., Bosworth, J.M., The international laser ranging service. Adv. Space Res., 30/2, 135-143. doi: 10.1016/S02731177(02)00277-6, 2002.

Reigber, C., Luhr, H., Schwintzer, P., CHAMP mission status. Adv. Space Res. 30 (2), 129-134, 2002.

Rothacher, M., Tapley, B.D., Reigber, C., et al., The tracking, occultation and ranging (TOR) instrument onboard TerraSAR-X and on TanDEM-X. In: Proceedings of International Geoscience and Remote Sensing Symposium IGARSS 2007, Barcelona, July 23-27, 4983-4986. doi: 10.1109/IGARSS2007.4423980, 2007.

RTCM, RTCM Standard 10403.1 with Amendment 1-5, Differential GNSS (Global Navigation Satellite Systems) Services - Version 3, Radio Technical Commission for Maritime Services, 1800 N. Kent St., Suite 1060, Arlington, Virginia 22209-2109, USA, July 1, 2011.

Schmid, R., Steigenberger, P., Gendt, G., et al. Generation of consistent absolute phase-center correction model for GPS receiver and antennas. J. Geod. 81 (12), 781-798, 2007.

Tapley, B.D., Bettadpur, S., Ries, J.C., et al. GRACE measurements of mass variability in the Earth system. Science 305 (5683), 503-505, 2004.

Tapley, B.D., Bettadpur, S., Watkins M.M. et al., The gravity recovery and climate experiment: mission overview and early results. Geophys. Res. Lett., 31, L09607. doi: 10.1029/2004GL019920, 2004b.

Warren, D.L.M., Raquet, J.F., Broadcast vs. precise GPS ephemerides : a historical perspective. GPS Solut. 7, 3, 151-156. doi: 10.1007/s10291003-0065-3, 2003.

Werninghaus, R., Buckreuss, S. The TerraSAR-X mission and system design. IEEE Trans. Geosci. Remote Sens. 48, 606-614, 2010.

Wu, S.C., Yunck, T.P., Theonton, C.L., Reduced-dynamic technique for precise orbit determination of low Earth satellites. J. Guid. Control Dynam. 14(1), 24-30. doi: 10.2514/3.20600. 1991.

Yoon, Y., Eineder, M., Yague-Martinez, N., et al., TerraSAR-X precise trajectory estimation and quality assessment. IEEE Trans. on Geosci. and Remote Sens., 47 (6), 1859-1868, 2009.

Zhang, X.H., Li, X.X., Guo, F., Satellite clock estimation at $1 \mathrm{HZ}$ for realtime kinematic PPP applications. GPS Solut. 15 (4), 315-324. doi: 10.1007/s10291-010-191-7, 2010. 